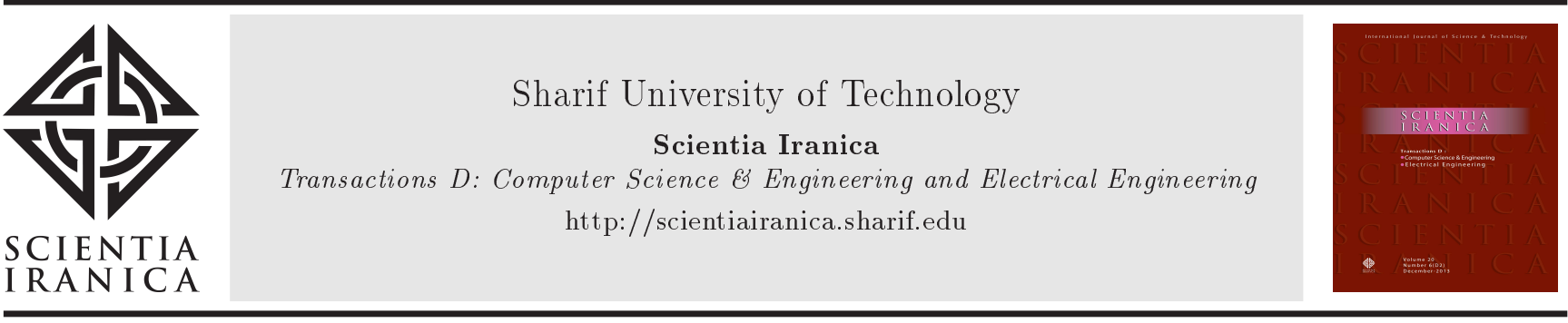

\title{
Gait generation and transition for bipedal locomotion system using Morris-Lecar model of central pattern generator
}

\author{
M.R. Sayyed Noorani ${ }^{a, *}$, S. Farshbaf Rashidi ${ }^{\mathrm{b}}$, and M. Shoaran ${ }^{\mathrm{b}}$ \\ a. Rehabilitation Robotics Research Lab., Department of Mechatronics Engineering, University of Tabriz, Tabriz, P.O. Box \\ 5166614761, Iran. \\ b. Department of Mechatronics Engineering, University of Tabriz, P.O. Box 5166614761, Tabriz, Iran.
}

Received 13 May 2016; received in revised form 16 January 2017; accepted 8 April 2017

\author{
KEYWORDS \\ Central Pattern \\ Generator (CPG); \\ Gait transition; \\ Bipedal locomotion; \\ Morris-Lecar \\ non-linear oscillator; \\ Genetic algorithm.
}

\begin{abstract}
In this paper, we intend to improve the CPG network presented by Pinto et al. based on 4-cell model for bipedal locomotion systems. This model is composed of four coupled identical cells and internal dynamics of each one is described by the Morris-Lecar nonlinear differential equation; also, the couplings between the cells follow the synaptic type. We exploited an elitist Non-dominated Sorting Genetic Algorithm (NSGA II) to find the best set of coupling weights by which the phase differences became optimally close to the ones required for a primary bipedal gait. Thus, we achieved the rhythmic signals associated with four primary bipedal gaits of walk, run, two-legged jump, and two-legged hop. Also, we successfully obtained all secondary gaits corresponding to the bipedal locomotion identified by Pinto et al. from the 4-cell model by symmetry breaking bifurcations of primary gaits. Particularly, we were able to produce the secondary gait, called "hesitation walk," through transition from primary gaits of run and two-legged jump.
\end{abstract}

(C) 2018 Sharif University of Technology. All rights reserved.

\section{Introduction}

In recent two decades, many researchers have studied locomotion patterns of different beings in order to imitate their locomotion mechanism in mobile robots. Humans and animals are capable of producing different locomotion patterns, which are called "gaits". Moreover, they are able to switch from one gait to another that is called "gait transition". They indeed can adjust to the environmental conditions by producing different gaits, e.g., walking in normal conditions, running in a

\footnotetext{
*. Corresponding author. Tel.: +984133393876 E-mail addresses: smrs.noorani@tabrizu.ac.ir (M.R. Sayyed Noorani); sahar.farshbaf@yahoo.com (S. Farshbaf Rashidi); mshoaran@tabrizu.ac.ir (M. Shoaran).
}

doi: $10.24200 /$ sci. 2017.4404 hurry, and jumping obstacles. Similarly, in robotics, the interest is in planning control strategies for legged robots to enable them to perform different gaits, as well as adaptively and robustly change the gaits $[1,2]$. These objectives have been considered in many recent researches on legged robots by using schemes that are inspired by biological neural networks called Central Pattern Generator (CPG). CPGs are the units of generation and transition of locomotion gaits in most of the animals and human. Some alternative bio-inspired methods can also be found in the literature, e.g., [3,4] that focused on the function of the musculoskeletal system to generate animal gaits. However, the CPGbased method is a neuro-musculo-skeletal approach that involves the neural architecture besides the musculoskeletal system to generate adaptive locomotion gaits for robots similar to naturalistic behaviors of animals. 


\subsection{Neurological background and developing CPG models}

In vertebrates, $\mathrm{CPG}$ unit is located in the spinal cord and its function is transforming the signals received from the high-level control center, i.e., brain, to stimulating neural oscillatory signals that are sent to muscles to perform a desired locomotion gait. In this process, the signal from the brain is only for activating the CPG network and is not oscillatory. Thus, producing neural oscillatory signals that make the musculoskeletal system perform the locomotion pattern asked by the brain is the task of the CPG. Specially, in order to produce these signals, there is no essential need for feedbacks from musculoskeletal system. That is, these signals are produced centrally in the pattern generator units. Nevertheless, sensorial information from environment could modulate the output rhythms of the CPG to achieve a stable locomotion gait [1].

The idea of the motor pattern generation by a unit not located in the brain was first introduced by Brown in 1911, by an experimental observation of a "decerebrated cat" $[5,6]$. In this study, the cat was stood on a mechanically driven conveyer belt, and despite the fact that it lacked cerebellum, it could walk almost naturally on the conveying belt. Even, the gait transitions to trot and gallop were respectively induced by slowly increasing the speed of the conveyer belt. These observations indicated that the unit of locomotion gait generator was not located at the upperlevel nerve centers such as cerebrum or cerebellum; rather they should probably be in the spinal nerve center.

Brown also presented a model for explaining how muscle stimulating neural oscillatory signals were produced based on the reciprocal inhibition principle, which is known as "half-center" model [5]. In Brown's model, units of rhythm generator produce alternating extension and flexion command signals. In detail, each unit includes two groups of neurons coupled mutually by inhibitory connections, having a fatigue mechanism. The output signals are sent to a pair of muscles that generate a rhythmic activity in musculoskeletal system. This conceptual model has been considered as a base for rhythmic pattern generation in locomotion systems of animals and humans.

The term "central pattern generator" was first introduced by Wilson when he was studying the locust flight motor patterns [7]. He showed that movement-related sensory feedback was not necessary for generating normal motor patterns, the discovery that was an evidence for the existence of a CPG for neurogenic flight in the locust [8]. Later, similar observations were reported by others. Grillner revealed that CPG networks were located in the spinal cord of vertebrates [5]. In fact, he showed that a cat could continue moving on a treadmill in spite of disconnection between its brain and spinal cord. Following this, Grillner and Cohen conducted researches on CPGs by studying lamprey locomotor system. They found out that electrical stimulation of a region in the brain stem, i.e., Mesencephalic Locomotor Region (MLR), could trigger the motion or change the motion speed [5,9].

The idea of central generation of motor neural signals attracted the attention of researchers and, consequently, several models were developed to describe it. Of the most important models are Hodgkin-Huxley (H-H), FitzHugh-Nagumo (FH-N), Morris-Lecar (ML), and Matsuoka leaky-integrator. It should be noted that these models are neurological. However, in engineering applications, CPG is a reference trajectory generator that produces periodic oscillatory signals. Thus, nonlinear oscillators such as phase or harmonic oscillators as well as relaxation oscillators are widely used to simulate CPGs [6].

It should be mentioned that developing the $\mathrm{CPG}$ models is important at least in two aspects; first, making predictions that lead to further development of physiological knowledge, and second, adaptively generating reference trajectories in robotic applications. The rhythmic trajectories produced by a CPG model can be used in driving multi-legged robots [10], or exploited in motion assist suits to accomplish synchronization and entrainment between periodic motions performed by human user and controlled by robot [11-12].

\subsection{Applications of CPG-based control in robotics}

From the viewpoint of robotics engineering, CPG networks consist in coupled oscillators each of which generates a reference trajectory for a joint of the kinematic chain of a robot. The outputs of these oscillators have the same frequency, but each one can take a phase shift from another. In fact, a set of phase differences can represent a particular locomotion gait. Thus, generating a desired gait requires adjusting the phase differences between joint trajectories. Adjusting the phase differences is achieved by tuning the weights imposed to control coupling strength of the oscillators. In this way, switching from one gait to another can result from a proper change in the coupling weights between oscillators. However, finding the correct coupling weights to generate a desired gait is a difficult task. Hence, some researchers focused on the design of architectures for CPGs constructed by coupled oscillators and in this way, succeeded to generate common gaits in legged robots such as bipeds [13-14] and quadrupeds [15-17], and even in legless robots such as fish [18] and snake robots [19-20].

Here, we briefly review some of the recent researches on gait generation and transition for legged robots that have applied CPG architectures as the motor pattern generator. $\mathrm{Wu}$ et al. adopted a $\mathrm{CPG}$ 
model in which phase differences between oscillators could be arbitrarily adjusted. They realized a continuous gait transition between walk and trot gaits in a quadruped robot. Specially, they used an adaptive frequency version of Hopf nonlinear oscillator as CPG, where its intrinsic frequency was adapted by sensory feedbacks [21]. Zhang et al. designed a fine trot gait for a parallel-leg quadruped robot named Baby Elephant. The gait generation was done based on what they called "series CPG" model. This model indeed represented a Fourier series for approximating the reference joint trajectories in which the Fourier coefficients were adaptively learned by Hopf nonlinear oscillators [22]. Liu et al. used CPGs for adaptive locomotion control of the AIBO quadruped and NAO biped robots, separately [23,24]. They applied Kimura's oscillators, a version of Matsuoka's neuron model, to online trajectory generation for reference points of the robots in workspace, rather than generating joint control signals in joint space. By this method, the number of oscillators and, consequently, their tuning parameters were reduced. It was also experimentally demonstrated that the trajectories were smoothly altered when the platform robots were walking up or walking down a slope. Cristiano et al. presented a 2layer CPG network to locomotion control of the NAO platform robot. This CPG network followed a masterslave topology, in which a central Matsuoka's oscillator was utilized to stimulate the oscillators placed at the head of the main chains attached to the robot's body. Then, active joints of the robot in each main chain were driven through slave oscillators associated with each of those joints. Also, in order to imitate the human gaits, some desired phase differences between the main chains of the robot were defined that should be met via adjusting the outputs of the slave oscillators. In addition, a Genetic Algorithm was used to find optimal parameters for the CPG network in openloop [25]. Likewise, Cristiano et al. drove the NAO robot to walk on unknown sloped terrains by a robust CPG network based on Matsuoka's oscillators that directly controlled the angular position of the robot's joints [26]. This was contrary to the method used by Liu et al. in [23,24]. Chen et al. investigated transitions between different gaits in a hexapod robot using a CPG including oscillators controlled by only one parameter to adjust the phase lags [27].

\subsection{Optimization method to design CPG networks}

As mentioned by Liu and Chen [28], parameter adjustment for CPG networks to generate the desired locomotion gait is a major difficulty. Besides the above-mentioned references, many others have tried to overcome this difficulty via applying search-based optimization methods. For example, Kim et al. used
Particle Swarm Optimization (PSO) to search the CPG parameters in order to generate optimal walk in a biped robot [29] and Oliveira et al. applied an Evolutionary Algorithm (EV) to search for the optimal set of the CPG parameters for stable walking in a humanoid robot [30].

Adjusting the parameters of a CPG model becomes more difficult when it is used to generate more than one gait. For example, human can perform gaits such as walk, run, jump, hop, etc. As a method for development of a CPG model that is able to produce various gaits, it can be useful to consider some symmetrical features existing in the movement patterns of legs or joints in the actual locomotor system.

\subsection{Bipedal gaits generation by the symmetric 4-cell CPG model}

It is observable that in primary bipedal gaits, the rhythmic movements of legs follow some symmetric patterns. For instance, in gaits of walk and run, the left and right legs move half period out of phase, while in two-legged jump and hop, the legs move in phase. Furthermore, if we focus on the flexor and extensor muscles of the ankle joints of the legs, we find out that they also behave with a symmetric phase pattern in activity. For instance, in the run, both flexor and extensor muscles of the ankle joints are in phase, whereas in the walk, they are half period out of phase. Researchers like Collins, Buono, Stewart, Golubitsky, and Pinto focused on such symmetries in animal gaits and developed a symmetrybased approach to investigate the global features of general classes of models for CPGs that could control bipedal, quadrupedal, and hexapodal locomotions [3134].

Recently, Pinto and Golubitsky investigated a 4cell model for CPG network architecture to produce rhythmic motor signals corresponding to bipedal gaits [33]. They predicted 11 possible periodic solutions to the 4-cell CPG model and classified them into two groups of primary and secondary gaits according to the waveform of the signals received by flexor and extensor muscles of the ankle joints. They could identify all four primary gaits, i.e., walk, run, two-legged jump, and two-legged hop, and four of six nontrivial secondary gaits, i.e., gallop, skip, asymmetric hop, and one-legged hop, with bipedal gaits actually performed by human. Later, another secondary gait was also identified that was called "hesitation walk". Then, Pinto et al. addressed gait transitions from a primary to a secondary gait in the 4-cell CPG model [34]. The gait transition was in fact the result of bifurcations occurring due to "symmetry-breaking" between couplings of the CPG network by varying certain parameters. It was also noted that the transitions were model-independent, i.e., they did not depend upon the mathematical model of the dynamics of the oscillators or the nature of 
the couplings. Pinto et al. examined the achieved gait transitions as well as both synaptic and diffusive couplings by two different models of Morris-Lecar and Wilson-Cowan oscillators [34].

\subsection{Contributions of this paper}

In this paper, we intend to follow the work of Pinto and Santos [34] in bipedal gaits generation and transition using the Morris-Lecar model of CPG. Particularly, we use a genetic algorithm with non-dominated sorting to search for optimal sets of the coupling weights in order to produce rhythms corresponding to the primary bipedal gaits of walk, run, two-legged jump, and twolegged hop. By exploiting this optimization method, we optimally adjust the phase differences locked between the rhythmic signals of the 4-cell CPG network as close as possible to what is actually desired. Also, we successfully obtain all secondary gaits corresponding to the bipedal locomotion identified by Pinto et al. from 4-cell model using symmetry breaking bifurcations of the primary gaits. Especially, we are able to produce the hesitation walk by transition from the primary gaits of run and two-legged jump. It should be mentioned that the hesitation walk has already been predicted by Pinto et al. as a possible periodic solution to 4-cell model; however, they did declare to be able to produce it by any bifurcation of the primary gaits, either from the run or from the two-legged jump [34].

The rest of this paper is organized as follows. Section 2 introduces the 4-cell CPG network, the phase-locking relations between the oscillators, and symmetries between couplings. Section 3 addresses the Morris-Lecar equations used for modeling the intrinsic dynamics of the cells. In Section 4, we briefly review a genetic algorithm with non-dominated sorting. Section 5 presents the results obtained by simulations based on the optimal coupling weights. Gait transitions from primary to secondary gaits, including the hesitation walk, are also shown. We draw some conclusions in Section 6 .

\section{Four-cell CPG network model}

The 4-cell CPG network model for bipedal gaits was firstly introduced by Golubitsky et al. [31], and then studied further by Pinto and Golubitsky [33]. As shown in Figure 1, this model consists of 4 identical cells each coupled with its adjacent cells via three connection ways that are horizontal (pairs of 1-2 and $3-4)$, vertical (pairs of 1-3 and 2-4), and diagonal (pairs of $1-4$ and $2-3$ ). It is well known that the model has a symmetric structure with respect to connections if two pairs of cells coupled through the same connection ways coordinately interchange their inter-connection signals as well as their weight values [34].

In the 4-cell CPG network model shown here, each

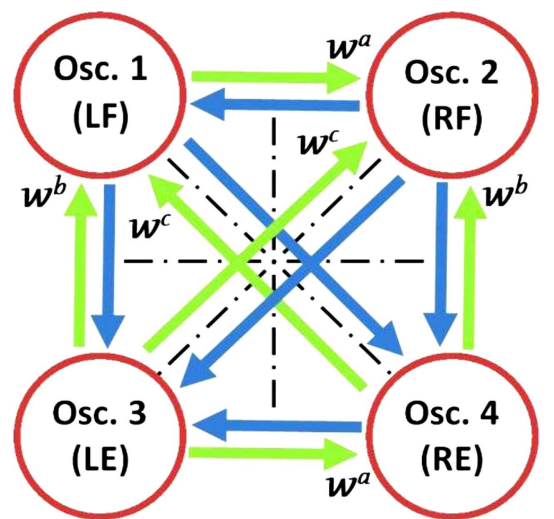

Figure 1. The four-cell CPG model. Oscillators produce the rhythmic signals sent to the flexor/extensor muscles of the left and right ankle joints. Coupling strength between the oscillators is controlled via three weights denoted by $w^{a}, w^{b}$, and $w^{c}$. Here, the blue arrows (that have no label) take the weight values equal to the ones given to the corresponding green arrows. However, secondary gaits may be produced when one of the symmetry ways is broken. Thus, weight values of two conjugated arrows are not equal necessarily. In the case of the secondary gaits, the arrows' weight values are denoted by a subscript " $i j$," where $i$ and $j$ determine the numbers of sending and receiving cells, respectively.

connection way is bilateral and one weight value controls the coupling strength in both directions equally. However, it should be noted that the equality is essential if a type of symmetry is required. A 4cell CPG model possesses a spatiotemporal symmetric structure wherever the wave shapes of the cell's output signals are the same. The primary gaits have this characteristic with respect to all symmetric directions. By definition, if all oscillators produce signals with the same waveform, whether in phase or out of phase, the resulting gait is recognized as a primary gait, otherwise it is secondary. The bipedal gaits of walk, run, two-legged jump, and two-legged hop are classified as primary gaits. In addition, a so-called spatial symmetry exists in the 4-cell CPG model whenever the output signals of two pairs of cells are also in phase. As shown in Figure 2, each primary gait possesses at least a spatial symmetry depicted by a symmetry line with respect to the corresponding cells and couplings. Specifically, the walk possesses the symmetry in diagonal way, the run has it in horizontal way, the two-legged jump has it in vertical way, and the two-legged hop has all types of these symmetries. Therefore, the 4-cell CPG model should possess all three ways of symmetry to be able to produce all primary bipedal gaits. In other words, generating the primary gaits is controlled by only three parameters of $w^{a}, w^{b}$, and $w^{c}$.

The architecture of the 4-cell CPG model has been inspired by biological reality. The cells placed at the 


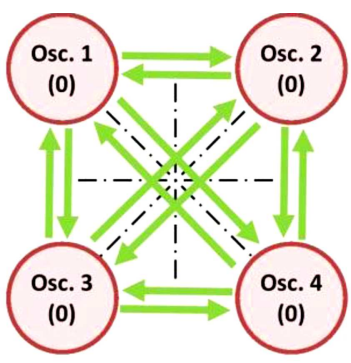

Two-legged hop

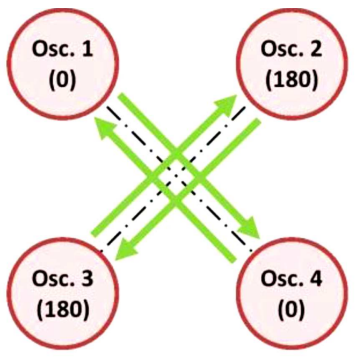

Walk

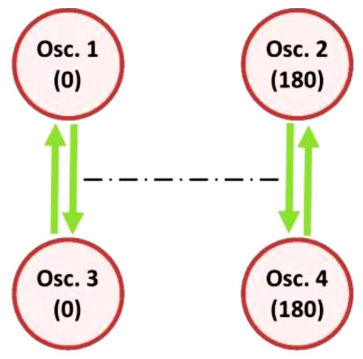

Run

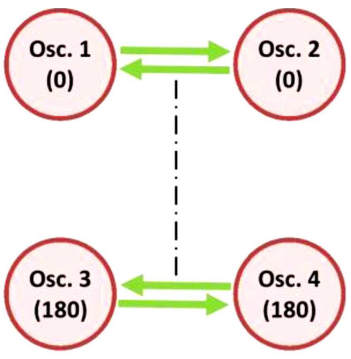

Two-legged jump

Figure 2. The locked phase differences should be held between the cells' output signals corresponding to the primary gaits. The symmetry lines depict the spatial symmetries existing in the primary gaits.

same column, see Figure 1, have roll of the flexor and extensor neurons associated with one leg. In fact, this is inspired by what happens in live musculoskeletal systems, where two muscle groups, namely, flexors and extensors, control the rhythmic movements of a joint. By these considerations, we are able to explain why the symmetric connections are required to adjust the phase differences between the cells in generating the primary bipedal gaits. Briefly, the symmetries are reflected in the CPG output rhythmic movements of the left and right legs as well as rhythmic activities of the ankle joints extensor and flexor muscles, the same as what happens in a live musculoskeletal system.

According to Figure 2, in the walk and run, the left and right legs are half period out of phase. However, in gaits of two-legged jump and hop, in which both legs move together uniformly, the left and right legs are always in phase. Hence, footprints of both legs are in the same row at the start and at the end of each jump or each hop. However, the ground contact duration in the two-legged jump is longer than that in the two-legged hop. Furthermore, in gaits of walk and two-legged jump, both flexor and extensor muscles of each leg are half period out of phase, because of the need for ankle rotation during performing the gaits. Contrariwise, in the run and two-legged hop, they are in phase, due to the need for rigidity in the ankle joints [32].

In the case of secondary gaits, first, the symmetry in the coupling ways considered in CPG architectures of primary gaits is broken, and consequently, because of the symmetry breaking, two different signals are produced by the cells. For example, the gallop may result from breaking of the symmetry in both the walk and two-legged jump gaits. Then, the muscle groups of the left leg receive the same signal shape, and those of the right leg receive another shape. Moreover, in both of the legs, the flexor and extensor muscles are half period out of phase. Therefore, the gallop has the symmetry neither in horizontal way of the two-legged jump nor in diagonal way of the walk.

\section{The Morris-Lecar nonlinear oscillator}

Various types of nonlinear oscillators have been presented to model the cells of a CPG [6]. The MorrisLecar model is one of them used in this paper because of its high resemblance to the biological model as well as simplicity. This model describes the rhythmic behavior of the cells of a CPG network by a set of nonlinear equations as follows:

$$
\left\{\begin{aligned}
\dot{u}= & f_{1}(u, v)=-\varphi \tau(v)(n(v)-u) \\
\dot{v}= & f_{2}(u, v)=-m(v) g_{c a}\left(v-V_{c a}\right) \\
& -g_{l}\left(v-V_{l}\right)-g_{k} u\left(v-V_{k}\right)+I_{a p p}
\end{aligned}\right.
$$

where, $v$ measures the membrane potential of neurons, $I_{\text {app }}$ is current stimulus, and each term $g_{c a}\left(v-V_{c a}\right)$, $g_{l}\left(v-V_{l}\right)$, and $g_{k}\left(v-V_{k}\right)$ models an ionic channel that regulates the voltage $v$ along the membrane of the axon. Also, the functions $m(v), n(v)$, and $\tau(v)$ are given by:

$$
\begin{aligned}
& m(v)=\frac{1}{2}\left(1+\tanh \left(\frac{v-V_{1}}{V_{2}}\right)\right), \\
& n(v)=\frac{1}{2}\left(1+\tanh \left(\frac{v-V_{3}}{V_{4}}\right)\right), \\
& \tau(v)=\cosh \left(\frac{v-V_{3}}{2 V_{4}}\right) .
\end{aligned}
$$

The remaining parameters are constant, chosen in numerical simulations as those given in Table 1 . The values of the parameters, except for $g_{c a}, \varphi$, and $I_{a p p}$, are fixed in all of the simulations.

\begin{tabular}{|c|c|c|c|c|c|c|c|c|c|c|c|}
\hline $\begin{array}{c}\boldsymbol{V}_{\mathbf{1}} \\
(\mathrm{mV})\end{array}$ & $\begin{array}{c}\boldsymbol{V}_{2} \\
(\mathrm{mV})\end{array}$ & $\begin{array}{c}\boldsymbol{V}_{3} \\
(\mathbf{m V})\end{array}$ & $\begin{array}{c}\boldsymbol{V}_{4} \\
(\mathbf{m V})\end{array}$ & $\begin{array}{c}\boldsymbol{V}_{k} \\
(\mathbf{m V})\end{array}$ & $\begin{array}{c}V_{l} \\
(\mathbf{m V})\end{array}$ & $\begin{array}{l}V_{c a} \\
(\mathbf{m V})\end{array}$ & $\begin{array}{c}\boldsymbol{g}_{k} \\
\left(\mathrm{mmho} / \mathrm{cm}^{2}\right)\end{array}$ & $\begin{array}{c}g_{l} \\
\left(\mathrm{mmho} / \mathrm{cm}^{2}\right)\end{array}$ & $\begin{array}{c}\boldsymbol{g}_{c a} \\
\left(\mathrm{mmho} / \mathrm{cm}^{2}\right)\end{array}$ & $\begin{array}{c}\boldsymbol{I}_{\mathrm{app}} \\
\left(\mu \mathrm{A} / \mathrm{cm}^{2}\right)\end{array}$ & $\varphi$ \\
\hline 0.01 & 0.15 & 0.10 & 0.15 & -0.70 & -0.50 & 1.00 & 2.00 & 0.50 & 0.90 & $0.23 \sim 0.30$ & $0.1 \sim 0.5$ \\
\hline
\end{tabular}

Eq. (1) describes the internal dynamics of one cell, while the output produced by the cell is a result

Table 1. The parameter values of the Morris-Lecar model used for numerical simulations. 
of coupling the cell with the other ones. Therefore, we should consider a model for couplings established between each two cells. Two models called synaptic and diffusive are introduced to this end [34]. Here, we choose the synaptic model that implies $h\left(y_{k}, y_{i}\right)=y_{k}$, where $h$ is coupling function, and $y_{k}$ and $y_{i}$ are the outputs of the deliverer and receiver cells, respectively. Accordingly, dynamics of the 4-cell CPG network model is formulated as follows:

$$
\begin{aligned}
\dot{y}_{1}= & F\left(y_{1}, y_{2}, y_{3}, y_{4}\right)=f_{2}\left(u_{1}, y_{1}\right)-w_{21}^{a} h\left(y_{2}, y_{1}\right) \\
& -w_{31}^{b} h\left(y_{3}, y_{1}\right)-w_{41}^{c} h\left(y_{4}, y_{1}\right) \\
\dot{y}_{2}= & F\left(y_{2}, y_{1}, y_{4}, y_{3}\right)=f_{2}\left(u_{2}, y_{2}\right)-w_{12}^{a} h\left(y_{1}, y_{2}\right) \\
& -w_{42}^{b} h\left(y_{4}, y_{2}\right)-w_{32}^{c} h\left(y_{3}, y_{2}\right), \\
\dot{y}_{3}= & F\left(y_{3}, y_{4}, y_{1}, y_{2}\right)=f_{2}\left(u_{3}, y_{3}\right)-w_{43}^{a} h\left(y_{4}, y_{3}\right) \\
& -w_{13}^{b} h\left(y_{1}, y_{3}\right)-w_{23}^{c} h\left(y_{2}, y_{3}\right), \\
\dot{y}_{4}= & F\left(y_{4}, y_{3}, y_{2}, y_{1}\right)=f_{2}\left(u_{4}, y_{4}\right)-w_{34}^{a} h\left(y_{3}, y_{4}\right) \\
& -w_{24}^{b} h\left(y_{2}, y_{4}\right)-w_{14}^{c} h\left(y_{1}, y_{4}\right),
\end{aligned}
$$

where, $y_{i}$ denotes the output of a cell. It should be noted that both $u_{i}$ and $y_{i}$ are variables of the cells' internal dynamics. Thus, according to Eq. (1), we have:

$$
\dot{u}_{i}=f_{1}\left(u_{i}, y_{i}\right) \quad i=1,2,3,4 .
$$

\section{Optimal primary bipedal gaits}

In generating the primary bipedal gaits, we should try to adjust the coupling weights so that cells' output signals possess phase differences similar to the ones considered in Figure 2. We solve this problem via a multiobjective optimization technique. In the first step, the objectives should be defined and formulated. It is clear that if each cell retains a correct phase difference from the cell numbered 1, then the phase difference between each two cells will be correct, too. Therefore, in the case of each primary gait, we formulate the objective functions as follows:

walk:

$$
\begin{aligned}
& z_{1}=\left|\Delta_{\phi}\left(y_{1}, y_{2}\right)-180^{\circ}\right|, \quad z_{2}=\left|\Delta_{\phi}\left(y_{1}, y_{3}\right)-180^{\circ}\right|, \\
& z_{3}=\left|\Delta_{\phi}\left(y_{1}, y_{4}\right)-0^{\circ}\right|,
\end{aligned}
$$

run:

$$
\begin{aligned}
& z_{1}=\left|\Delta_{\phi}\left(y_{1}, y_{2}\right)-180^{\circ}\right|, \quad z_{2}=\left|\Delta_{\phi}\left(y_{1}, y_{3}\right)-0^{\circ}\right| \\
& z_{3}=\left|\Delta_{\phi}\left(y_{1}, y_{4}\right)-180^{\circ}\right|
\end{aligned}
$$

Two-legged jump:

$$
\begin{aligned}
& z_{1}=\left|\Delta_{\phi}\left(y_{1}, y_{2}\right)-0^{\circ}\right|, \quad z_{2}=\left|\Delta_{\phi}\left(y_{1}, y_{3}\right)-180^{\circ}\right| \\
& z_{3}=\left|\Delta_{\phi}\left(y_{1}, y_{4}\right)-180^{\circ}\right|
\end{aligned}
$$

Two-legged hop:

$$
\begin{aligned}
& z_{1}=\left|\Delta_{\phi}\left(y_{1}, y_{2}\right)-0^{\circ}\right|, \quad z_{2}=\left|\Delta_{\phi}\left(y_{1}, y_{3}\right)-0^{\circ}\right|, \\
& z_{3}=\left|\Delta_{\phi}\left(y_{1}, y_{4}\right)-0^{\circ}\right|,
\end{aligned}
$$

where, $z^{\prime}$ s denote the objective functions and $\Delta_{\phi}(.,$. measures the phase differences between arguments via cross correlation analysis.

The elitist Non-dominated Sorting Genetic Algorithm (NSGA-II), introduced by Deb et al. [35], is utilized here to solve the multi-objective optimization problem formulated by Eqs. (4) for each primary bipedal gait. The optimizing variables are $w^{a}, w^{b}$, and $w^{c}$, by considering that the symmetries in all couplings should be conserved in the network. These variables are coded into a chromosome. An initial population is generated and in a repetitive procedure using the operators of selection, crossover, and mutation, new populations with higher fitness are generated. The NSGA-II ranks the populations according to a dominance rule. In this way, each solution of a population is assigned a fitness value based on its rank in the population, not its actual objective function value. Thus, these dummy fitness values determine an entire Pareto optimal solution set. A Pareto optimal set is a set of solutions that are nondominated by each other [36].

It is noted that, based on the dominance rule, solution $\mathbf{X}$ dominates $\mathbf{Y}$ if $\mathbf{X}$ is not worse than $\mathbf{Y}$ in any aspect (by evaluating objective functions) and $\mathbf{X}$ is better than $\mathbf{Y}$ at least in one facet. Hence, for each solution, a dominated set and a failure counter are assigned. Then, if $\mathbf{X}$ dominates $\mathbf{Y}$ in a comparison, $\mathbf{Y}$ is added to the dominated set of $\mathbf{X}$, and the failure counter of $\mathbf{Y}$ is increased by one. Thus, Pareto optimal solution set of rank of 1 includes the solutions with a failure count of zero. Other Pareto fronts are formed by repeating this for the remaining solutions with a failure count of 1 and so on. Eventually, a new population is generated from the best non-dominated fronts by regarding the crowding distance approach .

\section{Results and discussion}

As mentioned before, there are two contributions to present in this section. The first is the generation of primary bipedal gaits by the 4-cell CPG model with optimal coupling weights. The next is the generation of secondary bipedal gaits, especially the hesitation walk, by means of symmetry breaking bifurcations of the 
Table 2. Values of optimal coupling weights, cell parameters, and initial conditions used in generating the primary gaits.

\begin{tabular}{|c|c|c|c|c|c|c|c|c|c|c|}
\hline \multirow{3}{*}{$\begin{array}{c}\text { Gait } \\
\text { Walk }\end{array}$} & \multirow{2}{*}{\multicolumn{3}{|c|}{$\begin{array}{c}\text { Optimal coupling } \\
\text { weights values } \\
\left(w^{a}, w^{b}, w^{c}\right)\end{array}$}} & \multirow{2}{*}{\multicolumn{3}{|c|}{$\begin{array}{l}\text { Cells parameters } \\
\text { values } \\
\qquad\left(g_{\mathrm{ca}}, I_{\mathrm{app}}, \varphi\right)\end{array}$}} & \multicolumn{4}{|c|}{$\begin{array}{c}\text { Initial conditions } \\
\text { values }\end{array}$} \\
\hline & & & & & & & & $\left(\begin{array}{l}L F \\
L E\end{array}\right.$ & $\left.\begin{array}{l}R F \\
R E\end{array}\right)$ & \\
\hline & $(0.588$ & 0.092 & $0.238)$ & (0.900 & 0.230 & $0.255)$ & $\left(\begin{array}{c}(0.280 \\
(-0.197\end{array}\right.$ & $\begin{array}{c}0.277) \\
0.159)\end{array}$ & $\begin{array}{c}(-0.197 \\
(0.280\end{array}$ & $\left.\begin{array}{c}0.159) \\
0.277)\end{array}\right)$ \\
\hline Run & $(0.153$ & -0.281 & $0.143)$ & $(0.900$ & 0.290 & $0.395)$ & $\left(\begin{array}{c}(0.280 \\
(-0.197\end{array}\right.$ & $\begin{array}{r}0.277) \\
0.159)\end{array}$ & $\begin{array}{c}(-0.197 \\
(0.280\end{array}$ & $\left.\begin{array}{c}0.159) \\
0.277)\end{array}\right)$ \\
\hline $\begin{array}{l}\text { Two-legged } \\
\text { jump }\end{array}$ & $(-0.171$ & 0.003 & $0.739)$ & $(0.900$ & 0.280 & $0.295)$ & $\left(\begin{array}{c}(-0.046 \\
(0.327\end{array}\right.$ & $\begin{array}{l}0.398) \\
0.253)\end{array}$ & $\begin{array}{l}(0.046 \\
(0.327\end{array}$ & $\left.\begin{array}{l}0.398) \\
0.253)\end{array}\right)$ \\
\hline $\begin{array}{c}\text { Two-legged } \\
\text { hop }\end{array}$ & (0.101 & -0.210 & $-0.109)$ & $(0.900$ & 0.280 & $0.295)$ & $\left(\begin{array}{l}(-0.046 \\
(-0.046\end{array}\right.$ & $\begin{array}{l}0.398) \\
0.398)\end{array}$ & $\begin{array}{l}(-0.046 \\
(-0.046\end{array}$ & $\left.\begin{array}{l}0.398) \\
0.398)\end{array}\right)$ \\
\hline
\end{tabular}

Table 3. Values of cells parameters and coupling weights corresponding to gait transitions.

\begin{tabular}{|c|c|c|c|c|c|c|c|c|}
\hline \multicolumn{2}{|c|}{ Transition } & \multicolumn{3}{|c|}{$\begin{array}{c}\text { Cells parameters } \\
\text { values }\end{array}$} & \multicolumn{3}{|c|}{$\begin{array}{c}\text { Primary coupling } \\
\text { weights values }\end{array}$} & \multirow{2}{*}{$\begin{array}{l}\text { Changed coupling weights } \\
\text { values for symmetry } \\
\text { breaking bifurcations of } \\
\text { primary gaits }\end{array}$} \\
\hline $\begin{array}{l}\text { Primary } \\
\text { gait }\end{array}$ & $\begin{array}{l}\text { Secondary } \\
\text { gait }\end{array}$ & &,$I_{a p p}$ & & & $v^{a}, \boldsymbol{w}^{b}, \boldsymbol{u}$ & & \\
\hline Walk & Skip & $(0.900$ & 0.270 & $0.105)$ & $(0.588$ & 0.092 & $0.238)$ & $w_{3 \rightarrow 1}^{b}=w_{4 \rightarrow 2}^{b}=+0.558$ \\
\hline Run & Skip & $(0.900$ & 0.265 & $0.275)$ & $(0.153$ & -0.281 & $0.143)$ & $w_{3 \rightarrow 2}^{c}=w_{4 \rightarrow 1}^{c}=-0.200$ \\
\hline Walk & Gallop & $(0.900$ & 0.270 & $0.295)$ & $(0.588$ & 0.092 & $0.238)$ & $w_{2 \rightarrow 1}^{a}=w_{4 \rightarrow 3}^{a}=-0.553$ \\
\hline $\begin{array}{l}\text { Two-legged } \\
\text { jump }\end{array}$ & Gallop & $(0.900$ & 0.280 & $0.495)$ & $(-0.171$ & 0.003 & $0.739)$ & $w_{1 \rightarrow 2}^{a}=w_{3 \rightarrow 4}^{a}=-0.011$ \\
\hline Run & $\begin{array}{l}\text { Hesitation } \\
\text { walk }\end{array}$ & $(0.900$ & 0.295 & $0.175)$ & $(0.153$ & -0.281 & $0.143)$ & $w_{2 \rightarrow 1}^{a}=w_{3 \rightarrow 4}^{a}=-0.292$ \\
\hline $\begin{array}{l}\text { Two-legged } \\
\text { jump }\end{array}$ & $\begin{array}{l}\text { Hesitation } \\
\text { walk }\end{array}$ & $(0.670$ & 0.280 & $0.195)$ & $(-0.171$ & 0.003 & $0.739)$ & $w_{2 \rightarrow 1}^{a}=w_{3 \rightarrow 4}^{a}=-0.421$ \\
\hline $\begin{array}{l}\text { Two-legged } \\
\text { hop }\end{array}$ & $\begin{array}{l}\text { One-legged } \\
\text { hop }\end{array}$ & $(0.900$ & 0.270 & $0.295)$ & $(0.101$ & -0.210 & $-0.109)$ & $w_{2 \rightarrow 1}^{a}=w_{4 \rightarrow 3}^{a}=-0.300$ \\
\hline $\begin{array}{c}\text { Two-legged } \\
\text { hop }\end{array}$ & $\begin{array}{c}\text { Asymmetric } \\
\text { hop }\end{array}$ & $(0.900$ & 0.270 & $0.155)$ & $(0.101$ & -0.210 & $-0.109)$ & $w_{2 \rightarrow 1}^{a}=w_{3 \rightarrow 4}^{a}=+0.359$ \\
\hline
\end{tabular}

primary gaits. The results presented here have been obtained by numerical simulations in MATLAB.

In Table 2, we present the optimal values for coupling weights calculated by the NSGA-II. This algorithm applies the 2-point crossover operator in which crossover and mutation rates are set to 0.8 and 0.03 , respectively. In addition, the non-fixed parameters in internal dynamics of the cells as well as values of initial conditions to solve the dynamics are given here. Note that symmetry conditions corresponding to each primary gait are regarded in initial valuing. Figure 3 shows output rhythmic signals of the cells obtained by numerical simulations for each one of the walk, run, two-legged jump, and two-legged hop gaits, respectively.

After generating all primary gaits, we could also produce five secondary bipedal gaits via symmetry breaking bifurcations imposed by changing some values of coupling weights in the network of a primary gait. They were previously identified as patterns for bipedal gaits each of which can be a possible rhythmic solution to 4-cell CPG model [33]. Figure 4 shows architectures of the model by which we can generate the secondary gaits. Also, in Figures 5 to 8, the cells' output rhythmic signals corresponding to each generated secondary gait are shown. In Table 3, the values used in numerical simulations have been listed in detail, too.

As shown in Figure 3, in each case of primary gaits, the shape of the cells' output signals is the same. The phase differences have also been achieved same as those expected and shown in Figure 2. For example, all the cells' output signals corresponding to the twolegged hop are in phase. On the other side, in the case of secondary gaits, two different patterns in the 


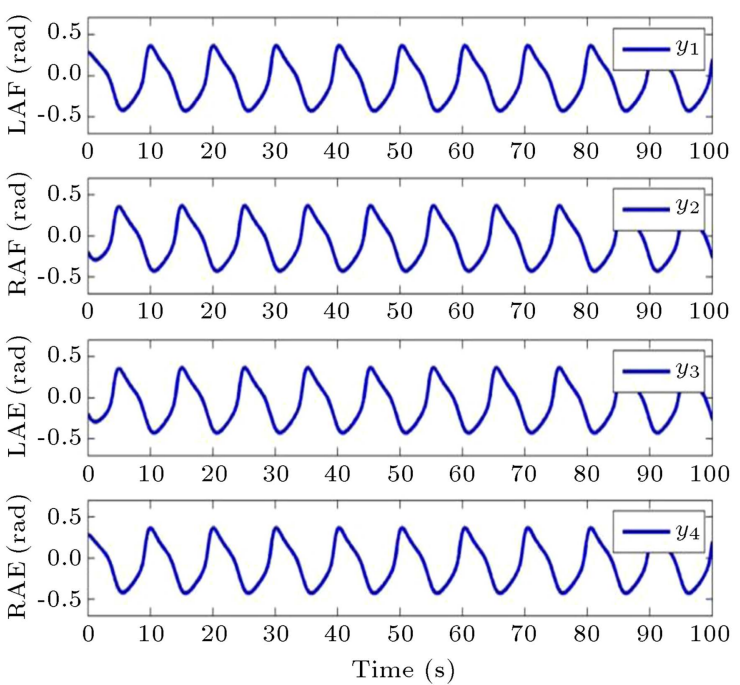

(a) Walk
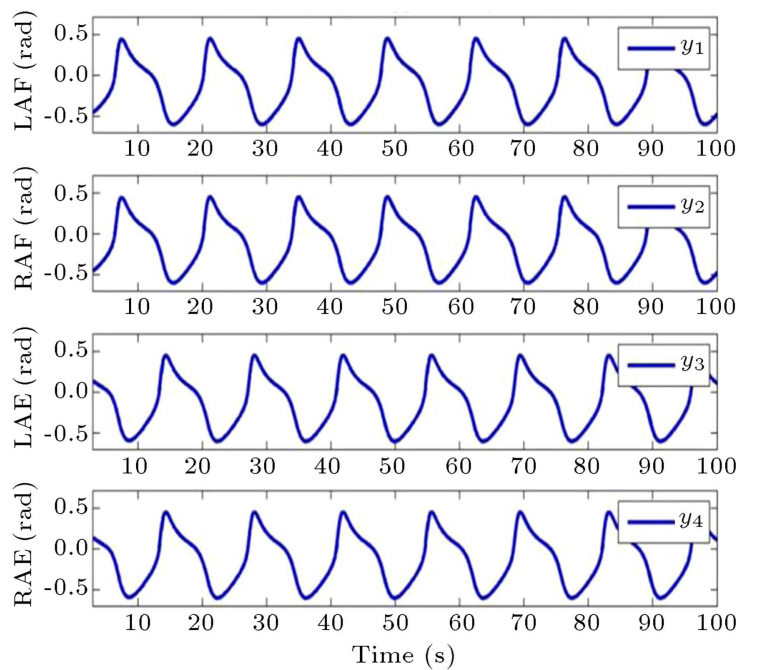

(c) Two-legged jump

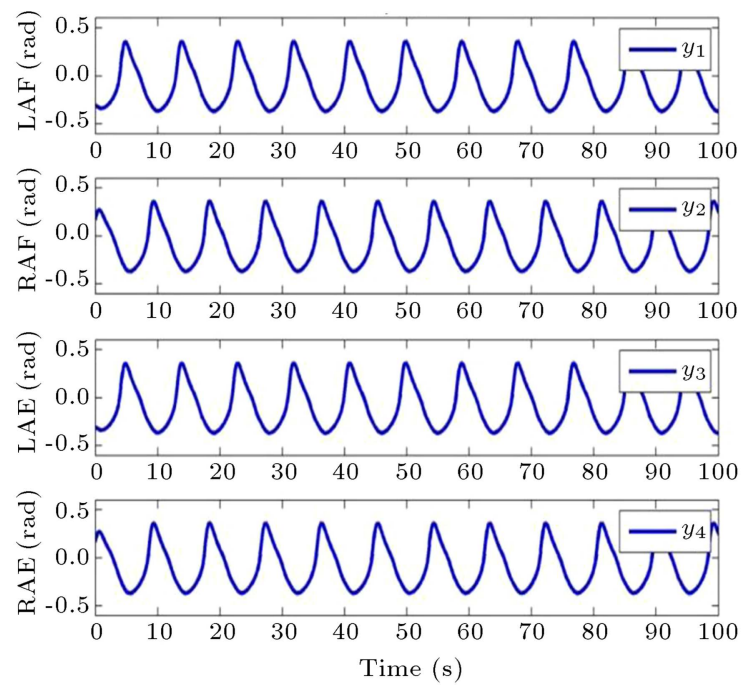

(b) Run

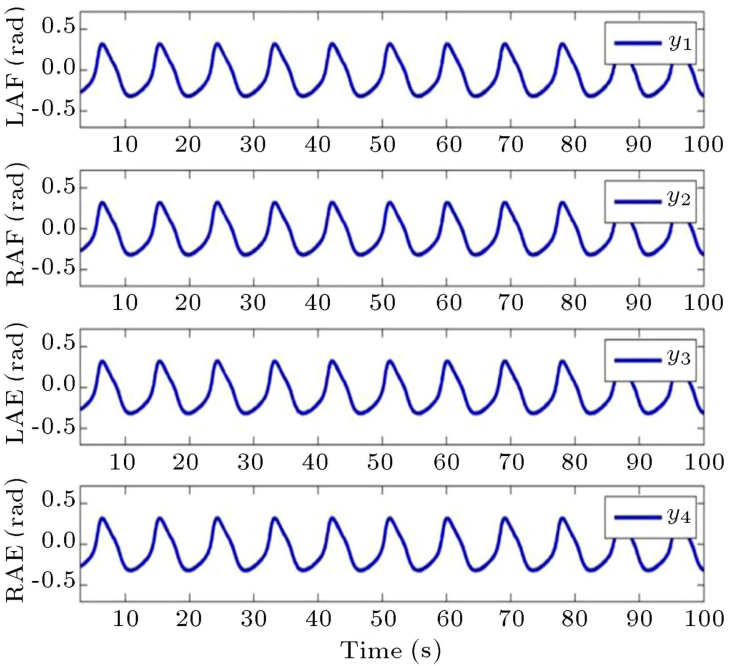

(d) Two-legged hop

Figure 3. The 4-cell rhythmic output signals corresponding to (a) walk, (b) run, (c) two-legged jump, and (d) two-legged hop.

output signals are revealed. We have shown each of the patterns with a distinct color in Figures 5 to 8 . It is clear that the output signals of secondary gaits do not possess any type of symmetry either with respect to patterns or by the way of phase lags. For example, in Figure 5(a), the output signals corresponding to the skip gait are shown, in which the pairs of cells that are adjacent vertically or opposite diagonally produce two different shapes of signals. Therefore, there is not any symmetry in horizontal or diagonal lines. Furthermore, the pairs of cells that are horizontally adjacent produce output signals with the same shape, but half period out of phase. Thus, there is no spatial symmetry in vertical way, too. However, it is possible that the signal patterns may coincide with each other via a shift along the time. Hence, it is said that a vertical spatiotemporal symmetry exists in the signals generating the skip gait.
Similarly, by paying attention to architectures shown in Figure 7, we can find a type of spatiotemporal symmetries for each secondary gait. The gallop and one-legged hop gaits have horizontal spatiotemporal symmetry; also, the hesitation walk and asymmetric hop have diagonal spatiotemporal symmetry. However, the secondary gaits generated by a transition from two-legged hop do not need to shift their same shape signals a long time to coincide with each other. Thus, a spatial symmetry exists here. Clearly, each spatial symmetry will also be a spatiotemporal symmetry. It is noted that the phase differences demanded between the cells of the networks in Figure 4 are meaningful when measured for signals of the same shape. By considering the cells' output signals shown in Figure 3, we find out that all primary gaits have all the three types of spatiotemporal symmetries. In addition, each one of the walk, run, and two-legged jump possesses only a 


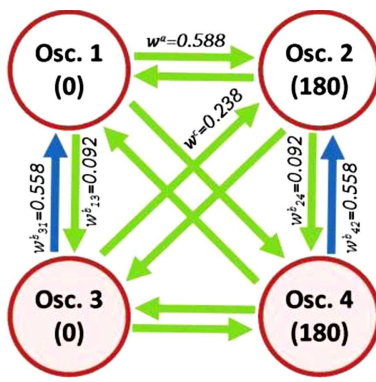

Walk to skip

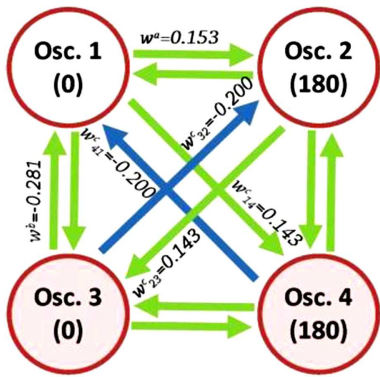

Run to skip

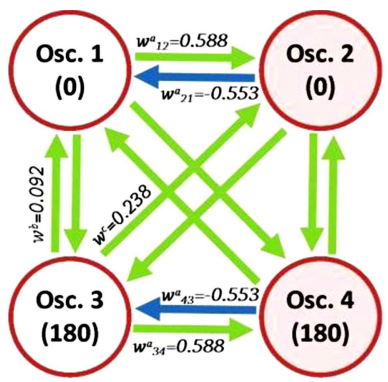

Walk to gallop

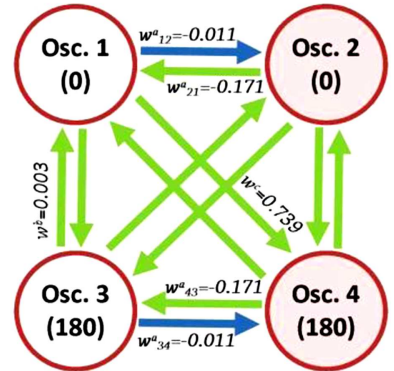

2-legged jump to gallop

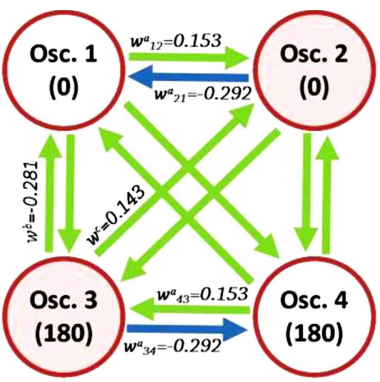

Run to hesitation walk

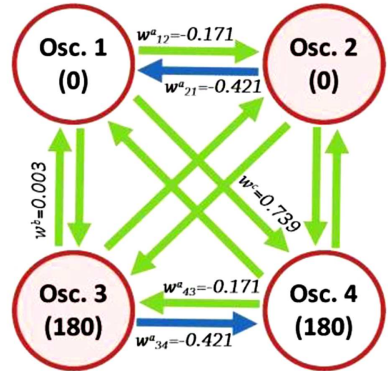

2-legged jump to hesitation walk

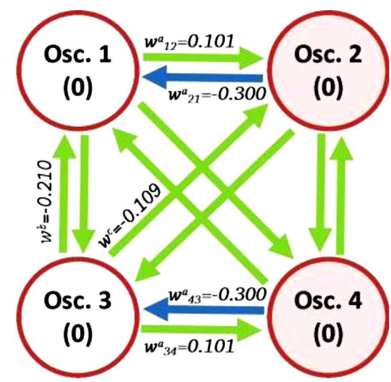

2-legged hop to 1-legged hop

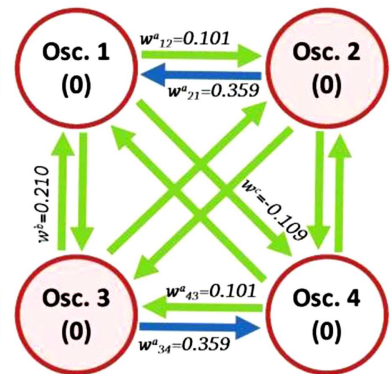

2-legged hop to asymmetric hop

Figure 4. Architectures of the 4-cell CPG network to generate secondary gaits via symmetry breaking bifurcation in a primary gait. In the secondary gaits of skip, gallop, and hesitation walk, each pair of signals having the same shape hold a phase lag of $180^{\circ}$. Thus, they have spatiotemporal symmetries. In contrast, in the cases of one-legged hop and asymmetric hop, signals of the same shape are in phase. Hence, they have some spatial symmetries.
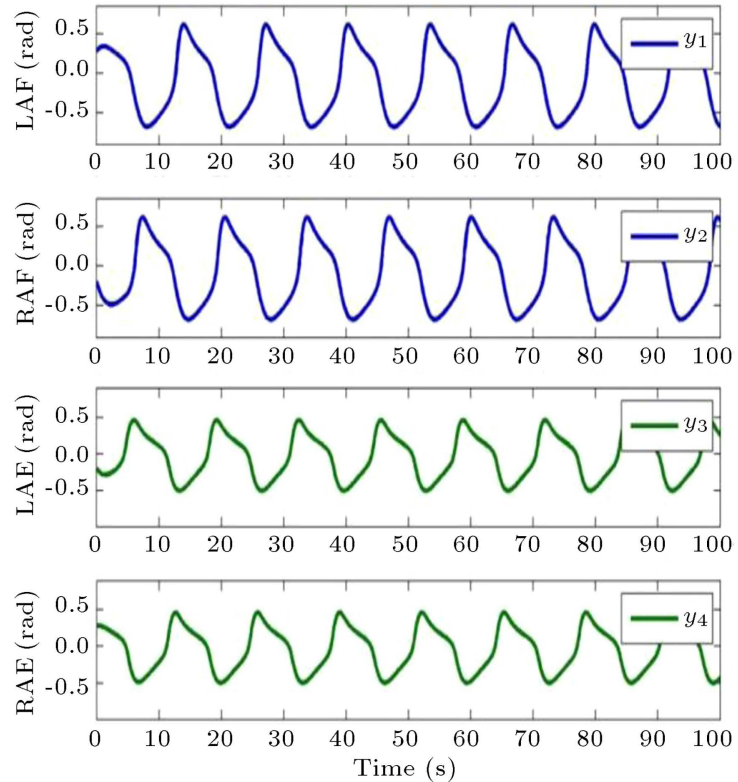

(a) Transition from walk to skip
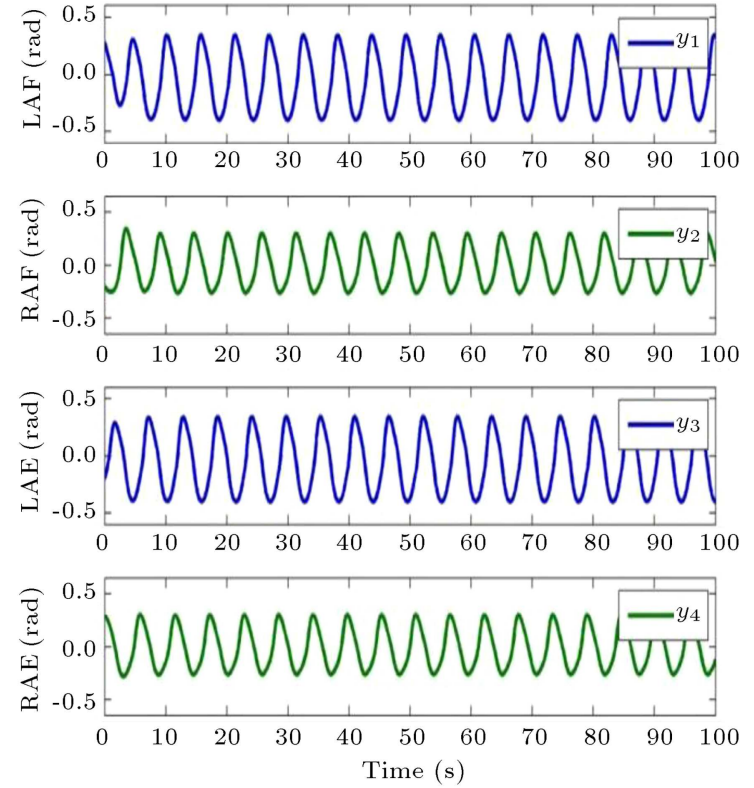

(b) Transition from walk to gallop

Figure 5. Transitions from (a) walk to skip and (b) gallop.

diagonal, a horizontal, and a vertical spatial symmetry, respectively. But, the two-legged hop possesses all types of the spatial symmetries.

As shown in Figure 4, each primary gait can produce two secondary gaits through transitions. Specially, we could produce the hesitation walk via transition from both the two-legged jump and the run. In both of the cases, it is achieved by changing the values of the horizontal coupling weights. Figures $6(\mathrm{~b})$ and 7(b) show two sets of signals producing the hesitation walk, in which the left flexor and the right extensor muscles receive signals of the same shape, however, half period out of phase. This is true for the left extensor and the right flexor, too; although, it does not mean 

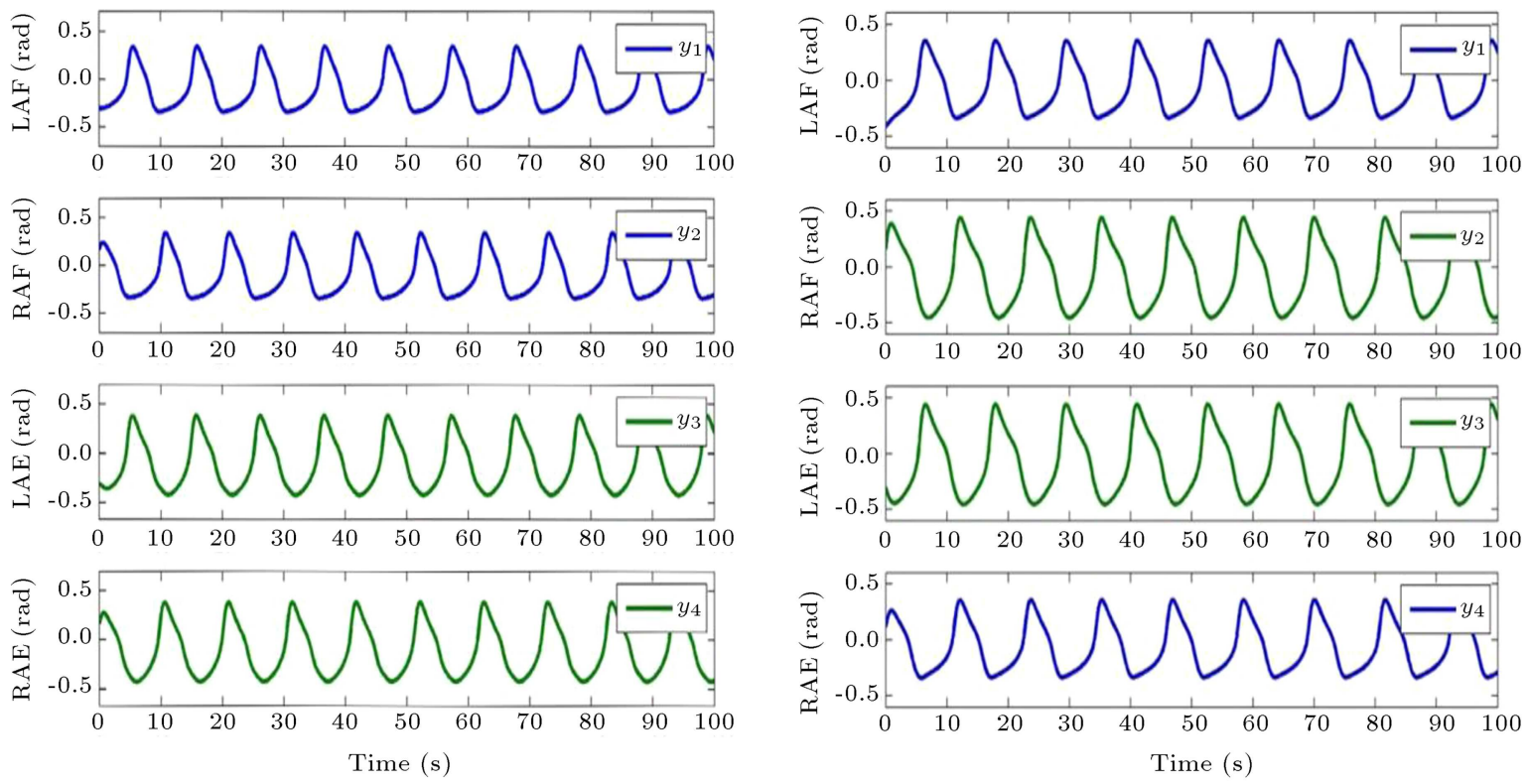

(a) Transition from run to skip

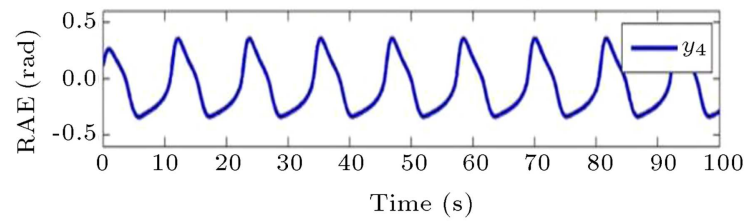

(b) Transition from run to hesitation walk

Figure 6. Transitions from (a) run to skip and (b) hesitation walk.
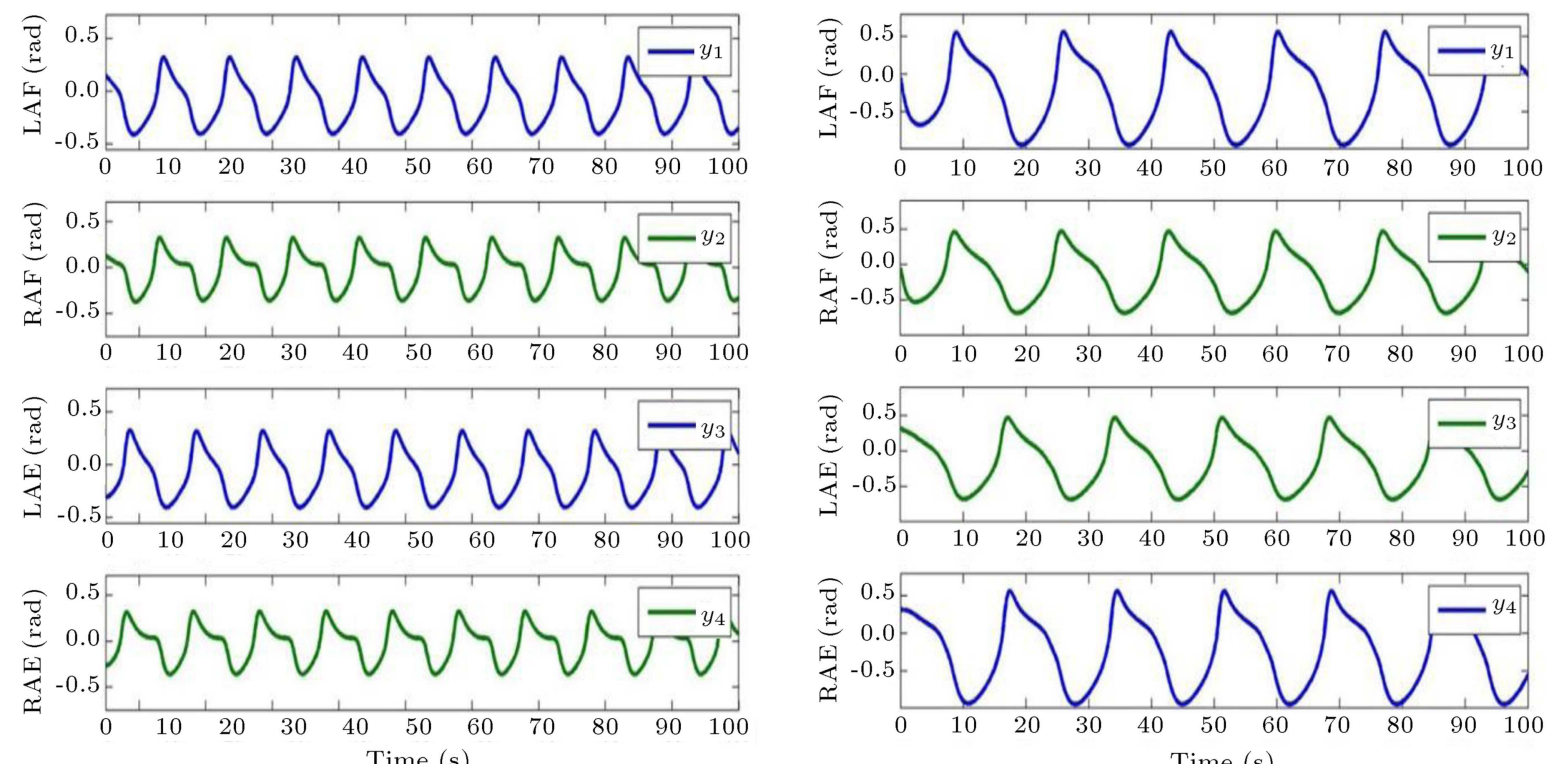

(a) Transition from 2 -L jump to gallop

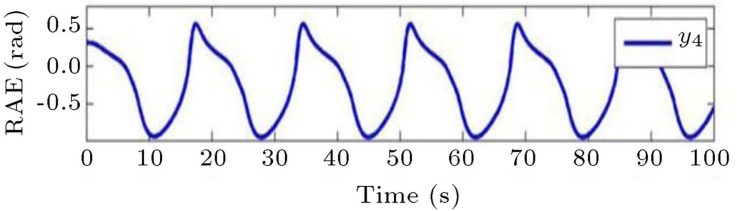

(b) Transition from 2 -L jump to hesitation walk

Figure 7. Transitions from (a) two-legged jump to gallop and (b) hesitation walk.

that there is an expected phase lag between the left and right extensors, or between the left and right flexors.

\section{Conclusions}

In this paper, we investigated the bio-inspired 4-cell CPG network model to generate rhythmic patterns corresponding to different primary bipedal gaits and transitions from them to secondary gaits. The model was composed of four coupled Morris-Lecar nonlinear oscillators jointed together via synaptic model of coupling, to generate rhythms sent to the flexor and extensor ankle muscles of the left and right legs. Existence of symmetries in establishing couplings drew out rhythmic output signals having all spatiotemporal symmetries and some spatial symmetries associated with the primary gaits. In order to adjust correct phase differences between the output signals by which we could be able to obtain the expected symmetries, a genetic algorithm with non-dominated sorting was used. Then, the secondary gaits were produced by symmetry breaking bifurcations of primary gaits caused by changing the values of some coupling weights in the network of primary gaits. The skip, gallop, 

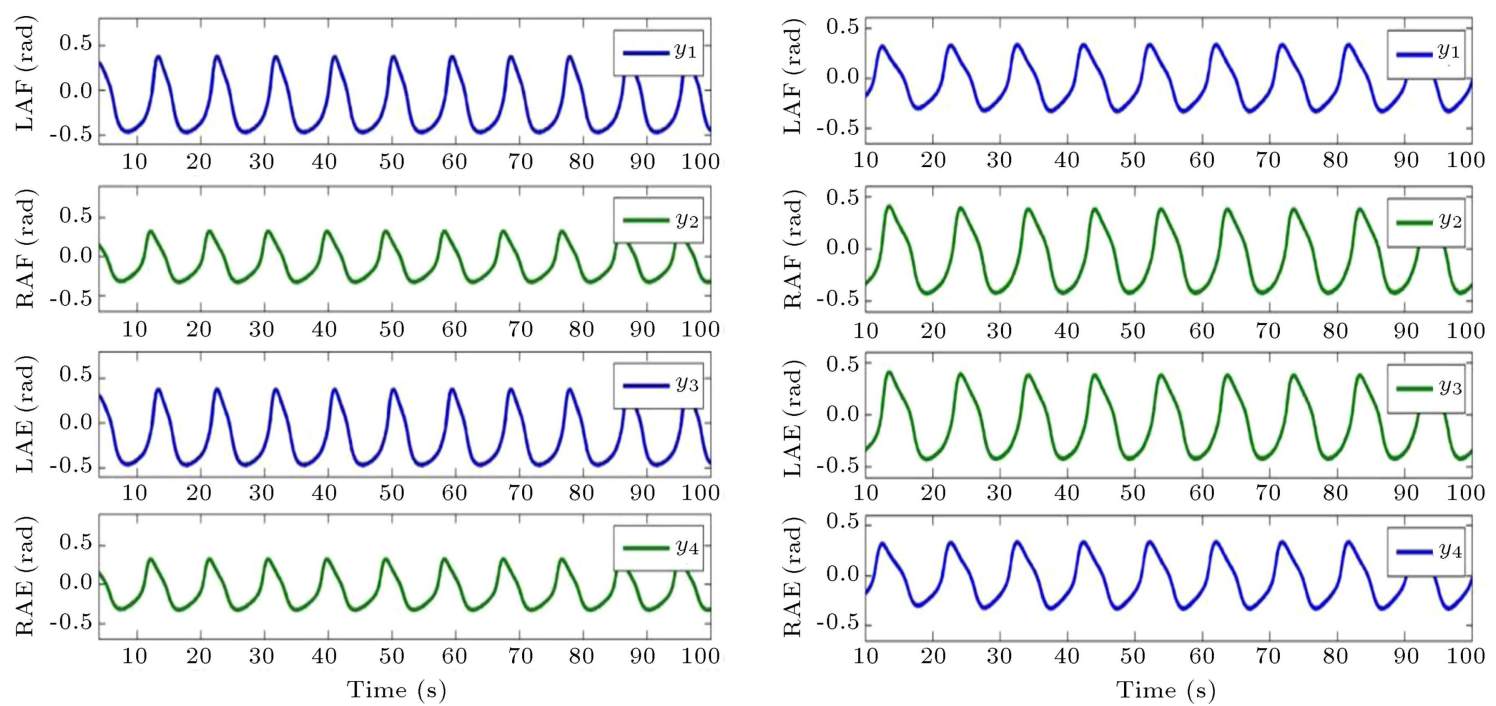

(a) Transition from 2-L hop to one-legged hop

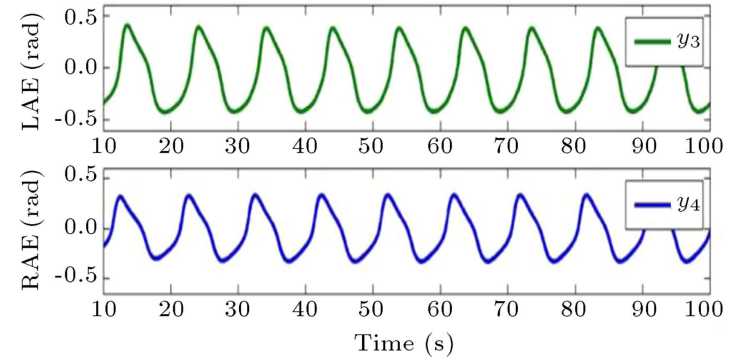

(b) Transition from 2-L hop to asymmetric hop

Figure 8. Transitions from (a) two-legged hop to one-legged hop and (b) asymmetric hop.

one-legged hop, asymmetric hop, and especially the hesitation walk gaits were produced by transitions from the primary gaits of walk, run, two-legged jump, and hop. Hesitation walk had not been obtained for run or Two-legged hop in [34]. It is worth to note that we recently presented a similar research paper in which diffusive model of coupling was examined to generate the secondary gaits [37]. However, we succeeded there in generating the hesitation walk only via transition from run.

\section{Nomenclature}

$v \quad$ Membrane potential

$I_{a p p} \quad$ Applied current stimulus

$g \quad$ Instantaneous membrane conductance

$v_{c a} \quad$ Equilibrium potential corresponding to $\mathrm{Ca}^{++}$

$v_{k} \quad$ Equilibrium potential corresponding to $\mathrm{K}^{++}$

$v_{l} \quad$ Equilibrium potential corresponding to leak

\section{References}

1. Semwal, V.B., Kumar, C., Mishra, P.K., and Nandi, G.C., IEEE Transactions on Automation Science and Engineering, 15(1), pp. 104-110 (Jan. 2018).

Semwal, V.B., Kumar, C., Mishra, P.K., and Nandi, G.C. "Design of vector field for different subphases of gait and regeneration of gait pattern", IEEE Transactions on Automation Science and Engineering, 15(1), pp. 104-110 (Jan. 2018).

2. Semwal, V.B., Mondal, K., and Nandi, G.C. "Robust and accurate feature selection for humanoid push recovery and classification: deep learning approach", Neural Computing and Applications, pp. 1-10 (2015).

3. Semwal, V.B., Katiyar, S.A., Chakraborty, R., and Nandi, G.C. "Biologically-inspired push recovery capable bipedal locomotion modeling through hybrid automata", Robotics and Autonomous Systems, 70, pp. 181-190 (2015).

4. Semwal, V.B. and Nandi, G.C. "Generation of joint trajectories using hybrid automate-based model: A rocking block-based approach", IEEE Sensors Journal, 16(14), pp. 5805-5816 (2016).

5. Ijspeert, A.J. "Central pattern generators for locomotion control in animals and robots: A review", Neural Networks, 21, pp. 642-653 (2008).

6. Wu, Q., Liu, Ch., Zhang, J., and Chen, Q. "Survey of locomotion control of legged robots inspired by biological concept", Sci. China. Ser. F-Inf. Sci., 52(10), pp. 1715-1729 (2009).

7. Wilson, D.M. "The central nervous control of flight in a locust", J. Exp. Biol., 38(47), pp. 471-490 (1961).

8. Marder, E. and Bucher, D. "Central pattern generators and the control of rhythmic movements", Current. Biology., 11, pp. 986-996 (2001).

9. Guertin, P.A. "The mammalian central pattern generator for locomotion", Brain research reviews, 62, pp. 45-56 (2009).

10. Aoi, S., Egi, Y., Sugimoto, R., Yamashita, T., Fujiki, S., and Tsuchiya, K. "Functional roles of phase resetting in the gait transition of a biped robot from quadrupedal to bipedal locomotion", IEEE Transactions on Robotics, 28(6), pp. 1244-1259 (2012).

11. Aguirre-Ollinger, G. "Exoskeleton control for lowerextremity assistance based on adaptive frequency oscillators: Adaptation of muscle activation and movement frequency", Proc. IMechE. Part H: J. Engineering in Medicine, 229(1), pp. 52-68 (2015). 
12. Zhang, X. and Hashimoto, M. "Evaluation on interaction ability of a walking robotic suit with synchronization based control", IEEE Int. Conf. Robotics and Biomimetics, Tianjin, China, pp. 265-27 (2010).

13. Taga, G. "A model of the neuro-musculo-skeletal system for human locomotion; I. Emergence of basic gait", Biological Cybernetics, 73, pp. 97-111 (1995).

14. Endo, G., Morimoto, J., Matsubara, T., Nakanishi, J., and Cheng, G. "Learning CPG-based biped locomotion with a policy gradient method: application to a humanoid robot", The International Journal of Robotics Research, 27(2), pp. 213-228 (2008).

15. Collins, J.J. and Richmond, S.A. "Hard-wired central pattern generators for quadrupedal locomotion", Biological Cybernetics, 71, pp. 375-385 (1994).

16. Fukuoka, Y., Kimura, H., and Cohen, A.H. "Adaptive dynamic walking of a quadruped robot on irregular terrain based on biological concepts", The International Journal of Robotics Research, 22(3-4), pp. 187202 (2003).

17. Buchli, J. and Ijspeert, A.J. "Self-organized adaptive legged locomotion in a compliant quadruped robot", Autonomous Robots, 25, pp. 331-347 (2008).

18. Crespi, A., Lachat, D., Pasquier, A., and Ijspeert, A.J. "Controlling swimming and crawling in a fish robot using a central pattern generator", Autonomous Robots, 25, pp. 3-13 (2008).

19. Wua, X. and Ma, S. "CPG-based control of serpentine locomotion of a snake-like robot", Mechatronics, 20, pp. 326-334 (2010).

20. Ryu, J-K., Chong, N.Y., You, B.J., and Christensen, H.I. "Locomotion of snake-like robots using adaptive neural oscillators", Intelligent Service Robotics, 3, pp. 1-10 (2010).

21. Wu, X., Teng, L., Chen, W., Ren, G., Jin, Y. and $\mathrm{Li}, \mathrm{H}$. "CPGs with continuous adjustment of phase difference for locomotion control", International Journal of Advanced Robotic Systems, 10, pp. 269-28 (2013).

22. Zhang, J., Gao, F., Han, X., Chen, X., and Han, X. "Trot Gait Design and CPG Method for a Quadruped Robot", Journal of Bionic Engineering, 11, pp. 18-25 (2014).

23. Liu, C., Chen, Q., and Wang, D. "CPG-inspired workspace trajectory generation and adaptive locomotion control for quadruped robots", IEEE Transactions on Systems, Man, and Cybernetics-Cybernetics, 41(3), pp. 867-880 (2011).

24. Liu, C., Chen, Q., and Wang, D. "Central Pattern Generator inspired control for adaptive walking of biped robots", IEEE Transactions on Systems, Man, and Cybernetics-Systems, 43(5), pp. 1206-1215 (2013).

25. Cristiano, J., Puig, D., and García, M.A. "Locomotion control of a biped robot through a feedback cpg network", in First Iberian Robotics Conference on Advances in Intelligent Systems and Computing, Springer International Publishing, Switzerland, pp. 527-540 (2014).
26. Cristiano, J., Puig, D., and Garcia, M.A. "Efficient locomotion control of biped robots on unknown sloped surfaces with central pattern generators", Electronics Letters, 51(3), pp. 220-229 (2015).

27. Chen, W., Ren, G., Zhang, J., and Wang, J. "Smooth transition between different gaits of a hexapod robot via a central pattern generators algorithm", J. Intell. Robot. Syst., 67, pp. 255-270 (2012).

28. Liu, C. and Chen, Q. "Methods synthesis of central pattern generator inspired biped walking control", in the 2015 Chinese Intelligent Automation Conference, Springer-Verlag, Berlin Heidelberg, pp. 371-379 (2015).

29. Kim, J.-J., Lee, J-W., and Lee, J.-J. "Central pattern generator parameter search for a biped walking robot using nonparametric estimation based particle swarm optimization", International Journal of Control, Automation, and Systems, 7(3), pp. 447-457 (2009).

30. Oliveira, M., Matos, V., Santos, C.P., and Costa, L. "Multi-objective parameter CPG optimization for gait generation of a biped robot", in IEEE Int. Conf. Robotics and Automation, Karlsruhe, Germany, pp. 3130-3135 (2013).

31. Golubitsky, M., Stewart, I., Buono, P.L. and Collins, J.J. "A modular network for legged locomotion", Physica D, 115, pp. 56-72 (1998).

32. Buono, P.L., and Golubitsky, M. "Models of central pattern generators for quadruped locomotion. I: Primary gaits", Journal of Mathematical Biology, 42, pp. 291-326 (2001).

33. Pinto, M. and Golubitsky, M. "Central pattern generators for bipedal locomotion", Journal of Mathematical Biology, 53(3), pp. 474-489 (2006).

34. Pinto, M. and Santos, A.P. "Modelling gait transition in two-legged animals", Communications in Nonlinear Science and Numerical Simulation, 16(12), pp. 46254631 (2011).

35. Deb, K., Agrawal, S., Pratap, A., and Meyarivan, T. "A fast elitist non-dominated sorting genetic algorithm for multi-objective optimization: NSGA-II", Lecture Notes in Computer Science, 1917, pp. 849-858 (2000).

36. Konaka, A.D., Coitb, W., and Smith, A.E. "Multiobjective optimization using genetic algorithms: A tutorial", Reliability Engineering and System Safety, 91, pp. 992-1007 (2006).

37. Farshbaf Rashidi, S., Sayyed Noorani, M.-R., and Shoaran, M. "Optimization of coupling weights in a 4-cell central pattern generator network for bipedal locomotion gait generation", Modares Mechanical Engineering, 16(12), pp. 144-152 (2016) (in Persian).

\section{Biographies}

Mohammad-Reza Sayyed Noorani received his BSc in Mechanical Engineering in 2006 from Sharif University of Technology, Engineering College in Golpayegan, Iran, and his MSc and $\mathrm{PhD}$ in Mechanical 
Engineering-Applied Mechanics-in 2009 and 2013, respectively, from University of Tabriz, Tabriz, Iran. At present, he is an Assistant Professor of Mechatronics Engineering at University of Tabriz. He is an associate member of the Iranian Society of Mechatronics. His research interests are in the areas of bio-inspired robotics, modeling and identification of dynamical systems, and especially rehabilitation robotics.

Sahar Farshbaf Rashidi received her BSc in Information Technology Engineering from University of Tabriz, Iran in 2012. She received her MSc in Mechatronics Engineering from School of EngineeringEmerging Technologies, University of Tabriz, Iran, in
2014. She is a member of the Iranian Society of Mechatronics. Her research interests include mechatronics and robotics.

Maryam Shoaran was born in Tabriz, Iran, in 1972. She received her BSc in Computer Engineering from Shahid Beheshti University, Tehran, Iran, in 2005. Also, she received the $\mathrm{MSc}$ and $\mathrm{PhD}$ degrees in Computer Science from University of Victoria (UVic), Canada, in 2007 and 2011, respectively. After 2 years of post-doctoral research at UVic, she came back to Iran. Currently, she is working as an Assistant Professor in the Department of Mechatronics Engineering at University of Tabriz. 